\title{
Applied Technology in 3D Geological Modeling of Oilfield based on Properties of Geological Materials a Review of Critical Issues3D
}

\author{
Xiaojun Liang \\ College of Earth Sciences \\ Jilin University \\ ChangchunP.R.China \\ e-mail:360154509@qq.com \\ Jinxin He \\ College of Earth Sciences \\ Jilin University \\ Changchun P.R.China \\ e-mail:jxhe@gmail.com
}

\author{
Laijun $\mathrm{Lu}$ \\ College of Earth Sciences \\ Jilin University \\ Changchun P.R.China \\ e-mail:ljlu@gmail.com \\ Tianyu Zhang \\ College of Earth Sciences \\ Jilin University \\ Changchun P.R.China \\ e-mail:tyzhang@gmail.com
}

\begin{abstract}
Oil acquisition and development is a difficult problem in the oil field and related geological study. The traditional geological exploration is of low accuracy, and need a lot of manpower, material and financial resources. The 3D geological modeling has been the foundation of oilfield development work and the modern science and technology performance. Thus, this paper mainly reviewed the critical issues of 3D geological modeling technology oriented to the oilfield. In summary, 3D geological modeling technology can reduce the error rate between the reservoir and the well. It can also improve the survey efficiency of reservoir which built the milepost of basic geological research of oilfield.
\end{abstract}

Keywords: Oil field; 3D geological modeling; Visualization technology

\section{I.INTRODUCTION}

The 3D geological modeling is a synthesis discipline based on data analysis and information analysis, or it is an integration of various disciplines. Such building model gathers

various information and interpretation of results.

In general, there are two kinds of methods related to geological information. A simulation and expression mainly adopts the profile and plan. Its essence is the three-dimensional space in the strata, tectonic, geomorphological and other geological phenomena of projection to a plane expression. With the aid of computer and science computing visualization technology, it is directly from three-dimensional perspective to understand and express the geological body and geological environment. It is an emerging discipline composed by geological exploration, mathematical geology, geophysics, mine geology, mine surveying, GIS, and visualization in scientific computing and other subjects. 3D geological models have important significance for engineering decision and scientific management: (1) It is helpful to improve the degree of information resources utilization and it is convenient to realize the system of management and resource sharing; (2)It is conducive to the realization of the analysis results of spatial expression and it is easy to enhance the visibility; (3) It uses the system method which integrate point and surface and the surface and the system method which integrate the regional planning and local areas. It is helpful to take quick, reasonable measures to reduce the cost as far as possible.

\section{THE STATUSOF 3D GEOLOGICAL MODELING}

The raise and development of three dimensional geological modeling theories is closely connected with the development of the geological statistics, while the development of the geological statistics is benefited from the research for kriging technique and the creative research for geological statistics. The 3D geological modeling was first proposed by scientists in Canada in 1993. But the theory of 3D modeling has a long history. Haldorson proposed the stochastic simulation of reservoir modeling method of dynamic reservoir simulation for oil scale in 1984. But the most representative of the modeling method of discrete smooth difference Mallet in 1989 and 1992 has published. It means the technology of three dimensional geological curved surface structure modeling technology of breakthrough. People have done a lot of research later, which mainly include the model and the structure of spatial data, the 3D visualization of data, the data structure of $3 \mathrm{D}$ vector map and so on. They have contributed to the development of the theory of three dimensional geological modeling.

\section{A. The principle of $3 D$ geological modeling.}

The principle of 3D model produced here is practical applicability, frugality and compatibility. Practical applicability means the required data in $3 \mathrm{D}$ model is 
different from the real goal, and different requirements to produce different 3D model. It will satisfy the above three aspects accurately, and analysis the geological data for geological exploration.

\section{B $3 D$ Geological Data Model.}

The spatial data model is the premise and basis to realize the 3D display and spatial analysis. Many scholars have done research on 3D spatial data model and data structure in recent years. Three data structures currently used for different space focus is different and also has not little difference in function. Three dimensional geological data model can be divided into data structure based on surface representation and data structure based on body representation, with the continuous deepening of the research, mixed data structure and object oriented data structure appeared.

\section{Visualization technology based on GIS.}

GIS is the abbreviation of Geographic Information Systems. The research of geography has developed quickly in the 1960s it is the product of traditional science and modern science and technology. Because GIS contains a large number of spatial geographic information and it can establish contract with the relevant data and information, using variation law of visualization results can be analyzed spatial property of the object. It is used in petroleum geological exploration, drilling and oil information management etc. Data structure based on surface representation is to describe and to represent the $3 \mathrm{D}$ body surface through the surface information. Its advantages are conductive to the display the geological body and the updated data. Its shortcoming is the lack of internal attributes of geologic body expression, so it is difficult to make spatial analysis of geological body. In these five kinds of structure, boundary representation is a representation of objects with regular shapes, and the other four ones are suitable for representing objects with irregular shape. The commonly used modeling methods of surface model based on a triangulated irregular network model, grid model, wire frame model, section model and so on. The data structure that is based on the body representation describes objects by the body information. As Fig. 1 shows, it takes the body element as the basic unit to represent the $3 \mathrm{D}$ entity. Due to the properties of element can be described independently and storage, it can carry out the 3D spatial operations and analysis of entity. However, it has the large storage space and the low calculation speed. The current commonly used modeling method based on body model are 3D grid, tetrahedral network, solid geometry structure, octree model, three prism and like the three prism model and so on.(1) Tin model triangulate the known data of scattered according to some kind of rules. Because it can be adapted to any complex surface, it has been widely used in surface modeling.

(2) B-rep is easy to describe the 3D object which structure is simple. It is inefficient that it cannot express the geological body which is irregular.

(3) TEN model is a 3D model of vector data structure. TEN has many advantages such as the structure of the body is simple, the geometric transformation is fast, the processing of topological relation is rapid and so on.

(4) Octree is a 3D grid model which the structure is improved. It describes an object hierarchically used tree structure. It is usually considered to be an uncommon primitive representation method. It is suitable for representing objects with complex shapes.

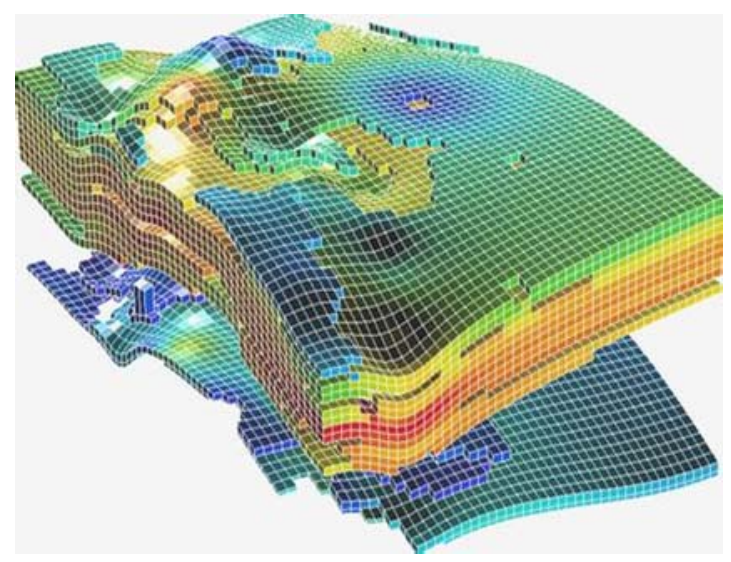

Figure.1: 3D visualization of oil field (Pictures from image.baidu.com)

\section{3D VISUALIZATION OF OIL FIELD}

Some sea oil surrounding environment is complex and changeable, and the formation characteristics is not outstanding. These lead to drilling power-off range cannot be accurately identified. This is one of the most difficult problems of oilfield development and oil acquisition. At present, the 3D visual technology used to oil development and oil collection. As Fig.2 shows, it can reduce the error rate between the reservoir and wells effectively. It not only improves the efficiency, but also improves the rate of oil reservoir survey simultaneously.

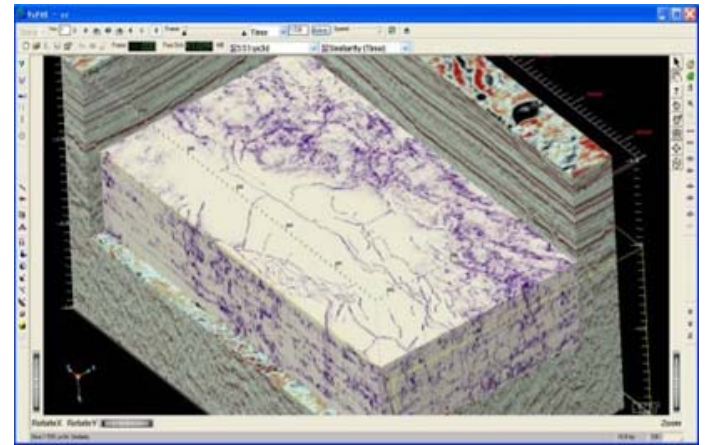

Figure.2: 3D visualization of oil (Pictures from image.baidu.com)

\section{A Digital oil storage.}

Firstly, drilling data, drilling position coordinates, drilling movement trace, measuring drilling curve, obtained from the text information and data seismic basic knowledge and so on the many kinds of relevant information and materials should be added in the computer when geological model is set up. Secondly, three dimensional geological modeling themselves also can generate a variety of information and data. As Fig.3 shows, these two aspects can obtain a complete information database. Three dimensional geological modeling has played a key role in the development of the whole oil and oil collection. An accurate model should know the underlying distribution, rock and sandstone thickness extent size data. It can improve the rate of oil reservoir survey and process a large amount of manpower, 
material and financial resources.

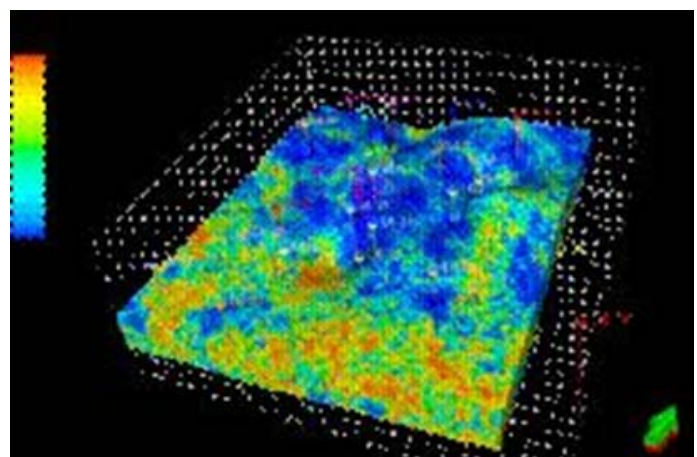

Figure.3: Digital oil storage of oilfield (Pictures from image.baidu.com)

\section{B Petroleum exploration.}

The spatial analysis function of visualization technology can be integrated with GIS technique uniquely and powerful visual expression ability in petroleum exploration field. It can link the underground geological structure, lithology, electrical property, oil characteristic, the ground exploration construction, and drilling engineering information to geographic information. Thus people can carry out exploration work deployment accurately, reasonably and quickly.

\section{Oil information management.}

The traditional oil information management systems focus only on their own scope of management. They cannot combine with other oil information management system effectively. Using visual technology and oil equipment of geographic space is a key index. It connects each system to attribute and spatial data application of equipment of each oil field. Its unified modeling management to the whole system of oil field makes the oil information management system be based on electronic map.

\section{$D$ The future trend of oil and gas modeling.}

In recent years, with the development of various type of oil reservoir exploration, it requires that people must understand distribution and geometry of space and time in sand under various environments. In particular, we must master the feature of single sand body geometry and 3D spatial continuity and the configuration of the relationship, and start modeling research in this area

A large number of three oil field experimental results of indoor tests so far less ideal in recent years. The reason is that the reservoir model is so different from the indoor simulation, and there may be the existing reservoir model is too rough or some geological factors are not found. With the increasing difficulty of exploration, it requires people to make a quantitative prediction to the physical parameters of reservoir. Only in the quantitative study of detailed first-hand information, and to find out the formation mechanism and a detailed analysis of mathematical statistics, it can be forecasted effectively as far as possible.

\section{Concluding remarks}

With the visualization technology of 3D geological modeling, we can describe the oilfield more clearly, solidly and really. Hence, this paper briefly reviewed the basic concept and the principles of 3D geological modeling, and described the 3D geological modeling in the oilfield. In conclusion, 3D geological modeling technology can reduce the error rate between the reservoir and the well, and also improve the survey efficiency of reservoir which built the milepost of basic geological research of oilfield.

\section{ACKNOWLEDGMENTS}

This work was supported by National 973 Basic Research Program of China (2015CB453000), Jilin Province Foundation of Applicable Sciences (20130102033JC) and Jilin Province Foundation of Youngster Scientists (20130522106JH). We also thank the anonymous reviewers for their helpful suggestions.

\section{REFERENCES}

[1] Ling Yonghong, Huang Xiaowei, “The 3D geological modeling technology and its realization in oilfield.” Computer Engineering pp. 96-98, 2009.

[2] Liu Bing, “The application of 3D geological technology in geological.Chinese petroleum and chemical standard and quality” 2012, pp. 225-227.

[3] Liu Jiyou,"The research and application of 3D geological body.”Daqing Petroleum Institute pp. 2004, 16-18.

[4] Ling Yonghong, Huang Xiaowei. “Oil of 3D geological modeling technology and software implementation.”Computer Engineering.2009 pp. 32-36.

[5] Dong Hui,“The geological body research on 3d visualization.” Central South University.2003 pp. 7-18 .

[6] LiQingyuan, Zhang Liyun, Wei Zhanying, Sun Liming"The 3D geological software research on the develop” Journals of Geology pp. 2013 55-57.

[7] HanJianguo, GuoDazhi, “The storage of octree and the information of ore body.”Journal of Surveying and mapping pp. 2002,33-35.

[8] Tang Zesheng,"The visualization of the data of 3D” Computer Engineering 1991, pp. 215-274.

[9] XUJiali,“The research of the visual data on the oil exploration and develop” Zhejiang University pp.2004 , 16-18

[10] Erich Grmma,“Design Pattern: Elements of Reusable Object-Oriented software” Addison-Wesley Longman Publishingpp. 2007.1-11.

[11]Richard. S. Wright, Benjamin Lipchak, Nicolas Haemel“OpenGL SuperbibleFourch Edition Sams” Indianapolis. 2007. pp. 24-28 .

[12]MatRuzinoorChe, Mahmud AhmadRodzi“Online 3D terrain visualization: A comparison of three different GIS software Quality" Engineering. 2009. pp. 483-487.

[13]Alan Petzet“Gains from Visualization” Computer science. 2002. pp. 17-33.

[14]Geoffrey A. Dorn,“Computing and Visualization” The Leading Edge. 2002 pp. 580-586. 